\title{
Clinical significance of recombinant RANBP2-ALK oncogene to radiotherapy in a case of locally advanced rectal epithelioid inflammatory myofibroblastic sarcoma: case report and review of literature
}

\author{
Cheng-Yen Lee ${ }^{1}$, Yu-Ling Hsu ${ }^{2}$, Ming-Yang Lee ${ }^{3}$, Chuan-Yin Fang ${ }^{4}$, Chien-Chin Chen ${ }^{5,6}$, Hsuan-Ying \\ Huang ${ }^{7}$, Yuh-Ru Liu ${ }^{8}$ \\ ${ }^{1}$ Department of Radiation Oncology, ${ }^{2}$ Department of Nuclear Medicine, ${ }^{3}$ Division of Hemato-oncology, ${ }^{4}$ Department of Colorectal Surgery, \\ ${ }^{5}$ Department of Pathology, Ditmanson Medical Foundation Chia-Yi Christian Hospital, Chia-Yi, Taiwan; ${ }^{6}$ Department of Cosmetic Science, Chia \\ Nan University of Pharmacy and Science, Tainan, Taiwan; ${ }^{7}$ Department of Anatomical Pathology, Kaohsiung Chang Gung Memorial Hospital and \\ Chang Gung University College of Medicine, Kaohsiung, Taiwan; ${ }^{8}$ Department of Nephrology, Ditmanson Medical Foundation Chia-Yi Christian \\ Hospital, Chiayi, Taiwan \\ Correspondence to: Yu-Ling Hsu, MD. Department of Nuclear Medicine, Ditmanson Medical Foundation Chia-Yi Christian Hospital, No. 539, \\ Zhongxiao Rd., East Dist., Chia-Yi 600, Taiwan. Email: cych07381@gmail.com.
}

\begin{abstract}
Epithelioid inflammatory myofibroblastic sarcoma (EIMS) is a highly aggressive variant of inflammatory myofibroblastic tumor (IMT). It is characterized immunohistologically by anaplastic lymphoma kinase (ALK) overexpression and genetic rearrangements in selected cases. The clinical significance of ALK rearrangement on radiotherapy was never reported in EIMS. Here we report a case of locally advanced rectal EIMS. In 2017, a 62-year-old woman was offered laparoscopic abdominoperineal resection for locally advanced EIMS of the rectum. Initial presentation was hematochezia and unintentional body weight loss. The resected tumor was positive for Ran-binding protein (RANBP2)-ALK fusion oncogene. Adjuvant radiotherapy was arranged for microscopic residual disease. Rapid intra-abdominal dissemination and local failure were identified shortly after the assigned treatments. She succumbed to the disease 134 days after diagnosis. In the era of precision oncology, the clinical significance of RANBP2-ALK recombination should be recognized. As treatment options are limited at time of treatment failure, upfront ALK inhibitor may be considered.
\end{abstract}

Keywords: Epithelioid inflammatory myofibroblastic sarcoma; Ran-binding protein 2; anaplastic lymphoma kinase (ALK); radiotherapy; case report; rectal cancer

Received: 17 July 2019; Accepted: 30 March 2020; Published: 30 June 2020.

doi: $10.21037 /$ tro.2020.04.01

View this article at: http://dx.doi.org/10.21037/tro.2020.04.01

\section{Introduction}

First described in the work by Marino-Enriquez, epithelioid inflammatory myofibroblastic sarcoma (EIMS) is an aggressive variant of inflammatory myofibroblastic tumor (IMT) with an epithelioid morphology and prominent inflammatory infiltrate (1). Although not ubiquitous, EIMS may be presented with anaplastic lymphoma kinase (ALK) gene rearrangements and are known to be associated with limited survival and rapid local recurrence (2).

Surgical excision and maximal debulking are considered standard for the treatment of EIMS. Systemic treatment with crizotinib, an ALK inhibitor, is reserved for salvage treatment and inoperable diseases (3-7). Radiotherapy is seldomly employed in the treatment of EIMS as most EIMSs are multifocal and arise on the mesentery and peritoneal surfaces in the abdominopelvic cavity. Of the 

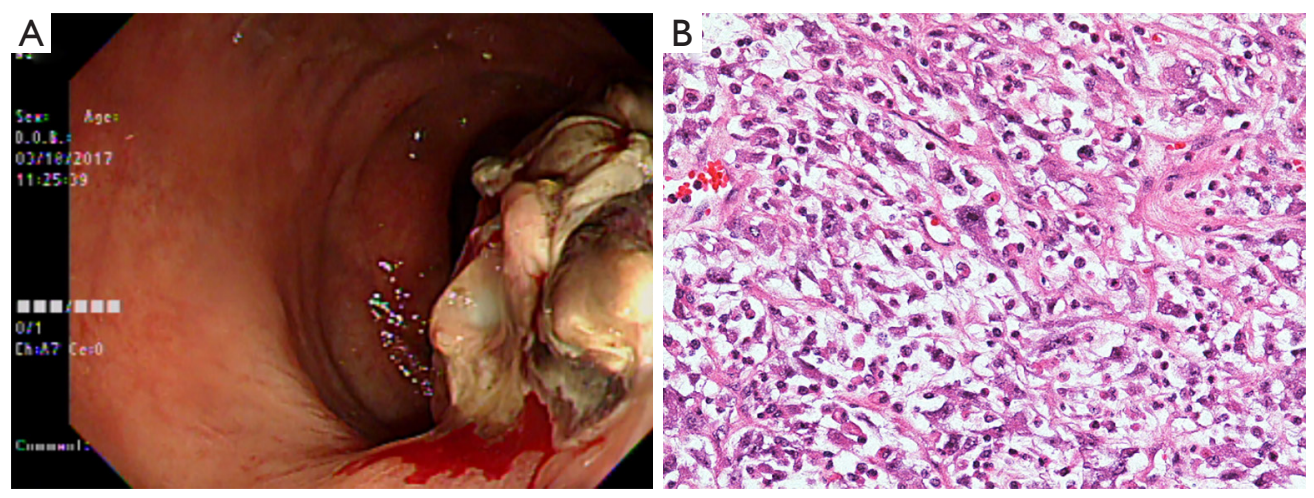

Figure 1 Gross and microscopic finding of the tumor. (A) Colonoscopy examination of EIMS presenting as a protruding polypoid mass in the rectal cavity. (B) Pathology with H\&E stain: the tumor composed of sheets of epithelioid cells in a myxoid and collagenous background (1/10 high power field).

28 reported EIMSs in English literature, only a handful cases with localized disease had radiotherapy; the effect of recombinant ALK oncogene on radiotherapy was never explored and should be taken into consideration in the era of precision oncology. We herein report on a case of ALK rearranged rectal EIMS, the only one of its kind, that had undergone surgical excision and adjuvant radiotherapy. This case is reported in accordance with the CARE guidelines. We present the following case in accordance with the CARE reporting checklist (available at http://dx.doi.org/10.21037/ tro.2020.04.01).

\section{Case presentation}

A 62-year-old Asian woman was admitted to our proctologist in March 2017 with an initial presentation of hematochezia, characterized by mixed fresh and clot blood. She had pale conjunctiva and frequent dizziness. Hemoglobin was $11.6 \mathrm{~g} / \mathrm{dL}$ at diagnosis. Body weight loss of $2 \mathrm{~kg}$ was noted in the preceding month. There was no fever, chillness, fullness, vomiting and poor intake. Relevant surgical history was total abdominal hysterectomy for uterine myoma ten years ago. Apart from that, she was on medication for hypertension. Digital rectal examination revealed a hard rectal mass just above dentate line. Endoscopic examination revealed an indurated polypoid mass (Figure 1A). Histologically, the tumor composed of sheets of epithelioid cells in a myxoid and collagenous background. Minor populations of spindled and plump polygonal ganglion-like myofibroblasts components were also identified. Mitotic figures (1/10 high power field) were observed. The tumor was infiltrated with neutrophils, lymphocytes and few plasma cells. At cellular level, the tumor cells had enlarged vesicular nucleoli and variable amounts of amphophilic cytoplasm (Figure 1B).

Combined ${ }^{18} \mathrm{~F}$-fluorodeoxyglucose positron emission tomography and CT $\left({ }^{18} \mathrm{FDG}-\mathrm{PET}-\mathrm{CT}\right)$ revealed a hypermetabolic mass in pelvis with maximal standard uptake value (SUVmax) at 13.3 (Figure 2, top row). She was offered laparoscopic abdominoperineal resection. At time of surgery, the tumor measured $10 \mathrm{~cm}$ in largest dimension. Extraluminal invasion to posterior wall of vagina was identified. No gross lymphadenopathy was noted. Postoperative recovery was uneventful. Immunohistochemically, the tumor cells were characteristic of ALK overexpression (nuclear membranous pattern). Ran-binding protein 2 (RANBP2) and ALK fusion was confirmed by reverse transcriptase-polymerase-chainreaction (RT-PCR) and sequencing assays (Figure 2, bottom row). In addition to involvement of deep circumferential margins, lymphovascular invasion was identified. None of the dissected 21 perirectal lymph nodes revealed evidence of metastatic tumor.

In the lack of government approval for ALK inhibitor in EIMS and gloomy evidence for conventional cytotoxic treatment, the multidisciplinary team decided to offer her adjuvant radiotherapy after surgery. Forty-five days after her surgery, radiotherapy without concurrent chemotherapy was offered to the patient. She was simulated in supine position with full bladder. Body markers were used to position the patient on treatment couch. Preoperative gross tumor volume (GTV) was reconstructed by registration the preoperative ${ }^{18} \mathrm{~F}-F D G$ PET-CT images to their simulation counterparts. The scans were transferred to, and registered 

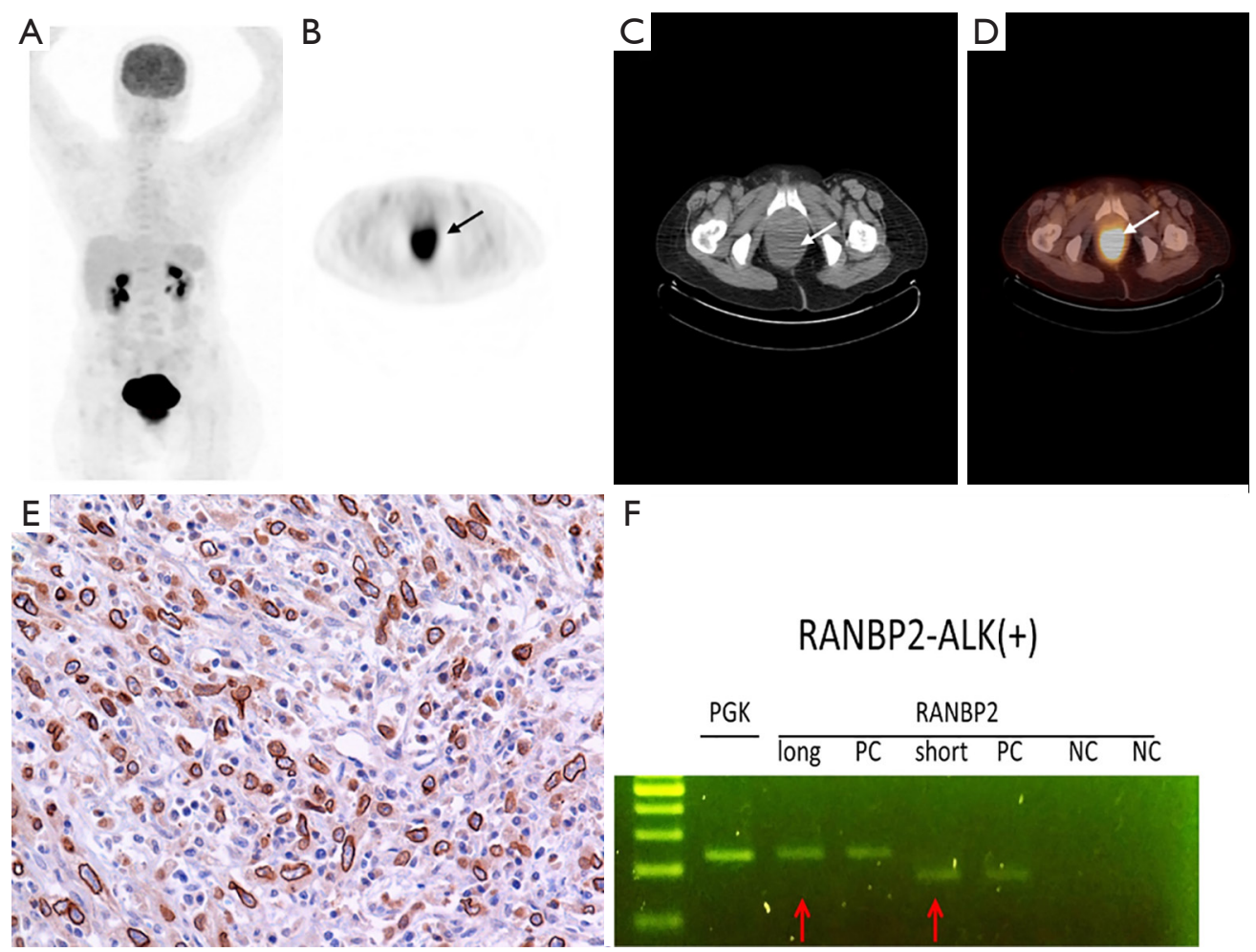

F

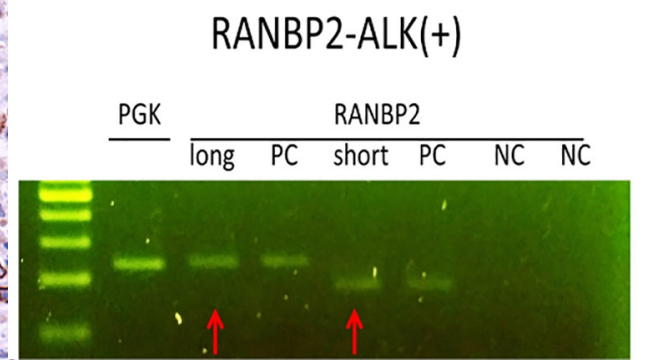

Figure 2 Top row: preoperative 18F-FDG PET/CT for the patient showed a hypermetabolic lesion in rectum (SUV max: 13.3). (A) Maximum intensity projection (MIP) image; (B,C,D) axial view of PET, CT, and PET/CT fusion. This PET/CT was warped onto simulation CT for treatment planning. Bottom row: (E) molecular examination of the resected tumor. Immunochemistry for ALK revealed a characteristic nuclear membrane pattern of staining (200x). (F) RANBP2-ALK fusion was detected by RT-PCR.

in the planning system (Eclipse A10, Palo Alto, USA). Manual target delineation for GTV was done using pathological findings and axial images with window level settings found most appropriate for this patient. The pelvic lymphatics were included for the presence of lymphovascular invasion. Intermediate risk clinical target volume (CTV-M) included the remaining rectum, mesorectum, and the internal iliac lymph nodes. The external iliac lymph nodes were also included because of invasion of urogenital space. The longitudinal extends of high risk clinical target volumes (CTV-H) included the parts of the remaining rectum that was within $2 \mathrm{~cm}$ longitude to the surgical anastomosis. Its circumferential extent of CTV-H included the mesorectal bed and terminated at uninvolved muscle, bone or air. Volumetric arc therapy in combination with imaged guidance was employed. CTV-M and CTV-H were treated to 4,600 and 6,600 cGy respectively (Figure 3A). In terms of side effect, the patient had Common Terminology Criteria for Adverse Events (CTCAE) grade 1 diarrhea and grade 2 moist desquamation. Radiotherapy was completed in
46 days, as scheduled.

She returned with abdominal fullness and diffuse abdominal pain 21 days after completing radiotherapy. Physical examination was compatible with massive ascites. In comparison to her treatment volumes at simulation, repeat ${ }^{18} \mathrm{~F}-F D G$ PET-CT showed bowel obstruction and advanced peritoneal seeding (Figure 3B). Residual disease in the surgical cavity was also visible (SUVmax: 33.0). Deterioration of her health was rapid. She succumbed to the disease 134 days after diagnosis.

\section{Discussion}

EIMS are very rare malignancies, with less than 30 cases reported in the English literature. To our knowledge, this patient is the only reported rectal EIMS with confirmed RANBP2-ALK fusion $(8,9)$. Multidisciplinary care remained the common approach to this condition, with surgery being the backbone of treatment. For localized rectal disease without RANBP2-ALK rearrangement, local tumor 

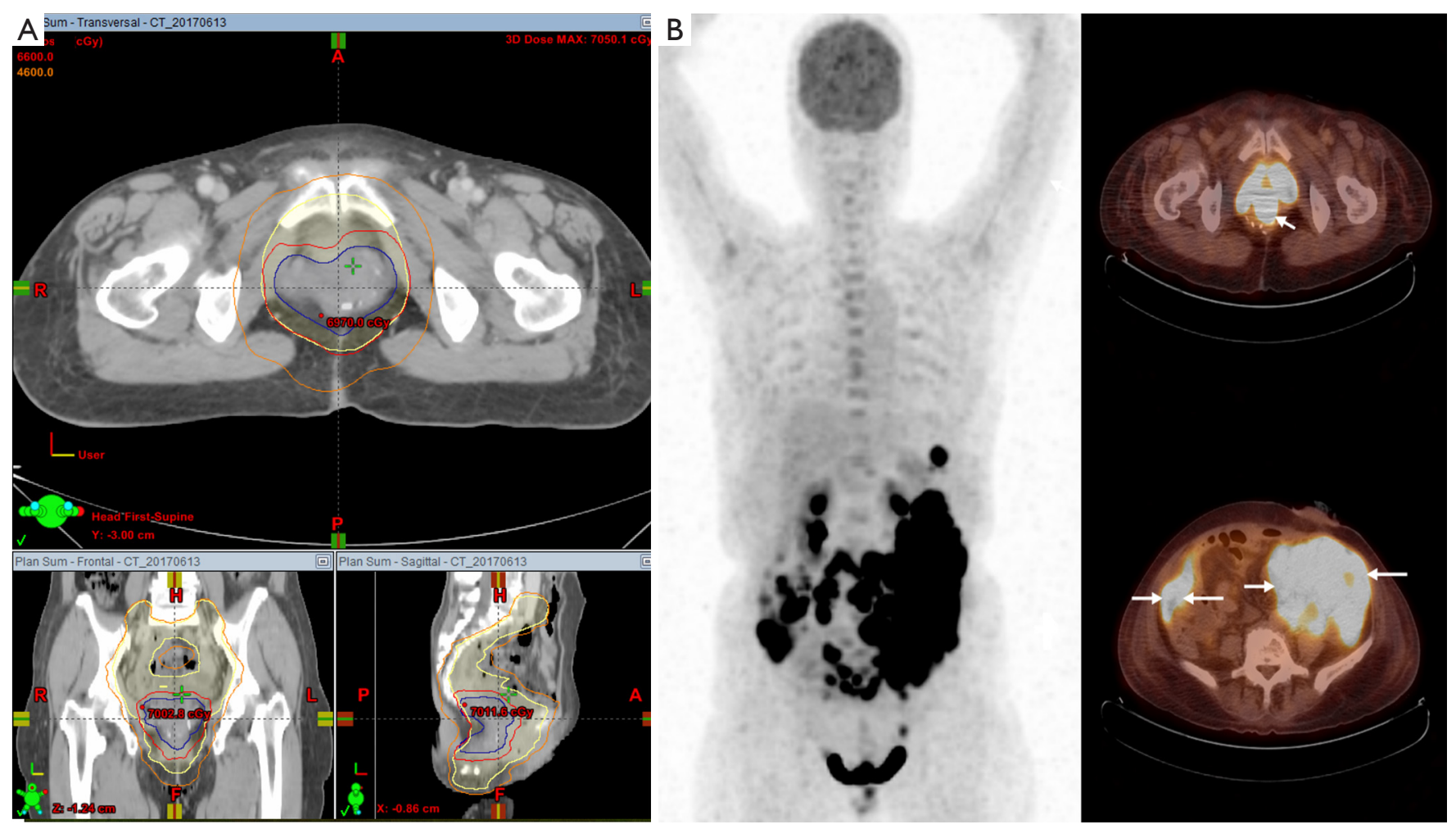

Figure 3 Comparison between treatment volume and 18F-FDG PET/CT at recurrence. (A) Treatment volumes on simulation CT. (B) Diffuse FDG uptake at abdomen and pelvic regions on MIP image was readily observable 44 days after completing radiotherapy. Axial view of PET, CT, and PET/CT fusion showed even higher FDG uptake at surgical bed (SUVmax: 33.0) along with peritoneal carcinomatosis (white arrows).

excision with partial protectomy alone provided months of local control (9). Even for multifocal diseases, surgical excision with maximal debulking remained the mainstay of primary treatment. It is advisory to have RANBP2-ALK rearrangement identified as part of surgical planning as it is predictive of both local recurrences and distant failure. Most oncogenic fusions occur at exon 18 of RANPB2 and exon 20 of ALK, and can be identified on RT-PCR (2). For our patient, microscopic breaches in the posterior peritoneal reflection may have led to subclinical intra-abdominal spread which eventually developed to diffuse peritoneal seeding. It is worth noting that immunohistochemical stain for ALK is neither prognostic nor predictive and should not be considered surrogate for RT-PCR (8).

Radiotherapy has been used in selected EIMSs and IMTs for the eradication of microscopic disease. Treatment outcome with radiotherapy was available for four patients (Table 1). In short, survival was much better for the previously reported cases; the longest survival was seen in a young male patient with disease in the sphenoid wing (8).
Of note, none of the previously reported cases had documented RANBP2-ALK fusion. The high SUVmax (Figure 3) value on repeat examination is indicative of high glucose transporter-1 expression, which is also indicative of poor prognosis and radioresistance among a variety of malignancies (10-13). Failure to accurately delineate the treatment volume is unlikely as the pre-treatment ${ }^{18} \mathrm{~F}-F D G$ PET-CT has been employed in the treatment planning. $6,600 \mathrm{cGy}$ is considered adequate for most mesenchymal tumors with microscopic disease.

Studies have not shown a consistent survival benefit to cytotoxic treatment, even administered intra-peritoneally with hyperthermia $(3,5,9)$. Certainly, for our case, she was too fragile for cytotoxic therapy at the time of disease progression. Targeted treatment, on the other hand, is more promising. Recombinant oncogene, although not universal for EIMS, presents an opportunity for targeted therapy. Crizotinib, an oral ALK inhibitor, was validated in multiple case reports at the time of disease relapse and treatment failure $(3-5,11,14)$. 
Table 1 Literature review of radiotherapy in the treatment of epithelioid inflammatory myofibroblastic sarcoma and inflammatory myofibroblastic tumors

\begin{tabular}{|c|c|c|c|c|c|c|c|c|c|}
\hline Case & $\begin{array}{l}\text { Age/ } \\
\text { gender }\end{array}$ & Site & $\begin{array}{l}\text { Size } \\
(\mathrm{cm})\end{array}$ & Pathology & $\begin{array}{l}\text { Multifocal at } \\
\text { diagnosis }\end{array}$ & Treatment & $\begin{array}{c}\text { Documented } \\
\text { RANBP2-ALK } \\
\text { fusion oncogene }\end{array}$ & Survival & Source \\
\hline 2 & $40 \mathrm{y} / \mathrm{M}$ & Peritoneum & 8 & EIMS & No & SE, ChT, RT & NA & DOD (28 m) & $\begin{array}{c}\text { Marino-Enriquez } \\
\text { et al. (10) }\end{array}$ \\
\hline 3 & $25 \mathrm{y} / \mathrm{M}$ & Sphenoid wing & 2 & IMT & No & SE, ChT, RT & NA & ANED (51 m) & Telugu et al. (6) \\
\hline 5 & $62 \mathrm{y} / \mathrm{F}$ & Rectum & 10 & EIMS & No & SE, RT & Yes & $\begin{array}{c}\text { DOD } \\
134 \text { days }\end{array}$ & Present case \\
\hline
\end{tabular}

ANED, alive with no evidence of disease; ChT, chemotherapy; DOD, died of disease; EIMS, epithelioid inflammatory myofibroblastic sarcoma; F, female gender, IMT, inflammatory myofibroblastic tumors; M, male gender, NA, not available; SE, surgical excision; RT, radiotherapy

Given the high rate of local recurrence and limited options of treatment after relapse, the combination of crizotinib and radiotherapy may be a valid option for adjuvant treatment for EIMS with RANBP2-ALK rearrangement. Moreover, crizotinib has been found to act synergistically with radiotherapy in lung cancer model, potentially improving local control in addition to its established systemic effect $(15,16)$. At the time of writing, crizotinib was approved by the FDA for use in metastatic non-small cell lung cancer patients whose tumors are ALK or ROS1 positive (17).

In conclusion, for EIMS, treatment results of radiotherapy varied significantly. Our study demonstrated that the combination of laparoscopic abdominoperineal resection and adjuvant radiotherapy may be an undertreatment for locally advanced rectal EIMS case with RANBP2-ALK rearrangement. Upfront ALKinhibitor should be employed to address both local control and subclinical disease in the abdominopelvic cavity, as treatment options are limited at time of disease progression.

\section{Acknowledgments}

We would like to express our gratitude to healthcare workers who were involved in the care of the patient.

Funding: None.

\section{Footnote}

Reporting Checklist: The authors have completed the CARE reporting checklist. Available at http://dx.doi.org/10.21037/ tro.2020.04.01

Conflicts of Interest: All authors have completed the ICMJE uniform disclosure form and declare (available at http:// dx.doi.org/10.21037/tro.2020.04.01). The authors have no conflicts of interest to declare.

Ethical Statement: The authors are accountable for all aspects of the work including full data access, integrity of the data and the accuracy of the data analysis. All procedures performed in studies involving human participants were in accordance with the Declaration of Helsinki (as revised in 2013). This study was approved by the institutional review board (No. 106098). The patient has died and thus is unable to provide consent. Consent to publish was taken from her daughter.

Open Access Statement: This is an Open Access article distributed in accordance with the Creative Commons Attribution-NonCommercial-NoDerivs 4.0 International License (CC BY-NC-ND 4.0), which permits the noncommercial replication and distribution of the article with 
the strict proviso that no changes or edits are made and the original work is properly cited (including links to both the formal publication through the relevant DOI and the license). See: https://creativecommons.org/licenses/by-nc-nd/4.0/.

\section{References}

1. Mariño-Enríquez A, Wang WL, Roy A, et al. Epithelioid inflammatory myofibroblastic sarcoma: An aggressive intra-abdominal variant of inflammatory myofibroblastic tumor with nuclear membrane or perinuclear ALK. Am J Surg Pathol 2011;35:135-44.

2. Li J, Yin WH, Takeuchi K, et al. Inflammatory myofibroblastic tumor with RANBP2 and ALK gene rearrangement: a report of two cases and literature review. Diagn Pathol 2013;8:147.

3. Butrynski JE, D'Adamo DR, Hornick JL, et al. Crizotinib in ALK-rearranged inflammatory myofibroblastic tumor. N Engl J Med 2010;363:1727-33.

4. Fang H, Langstraat CL, Visscher DW, et al. Epithelioid Inflammatory Myofibroblastic Sarcoma of the Ovary With RANB2-ALK Fusion: Report of a Case. Int J Gynecol Pathol 2018;37:468-72.

5. Kimbara S, Takeda K, Fukushima H, et al. A case report of epithelioid inflammatory myofibroblastic sarcoma with RANBP2-ALK fusion gene treated with the ALK inhibitor, crizotinib. Jpn J Clin Oncol 2014;44:868-71.

6. Ogata M, Hatachi Y, Ogata T, et al. Effectiveness of Crizotinib for Inflammatory Myofibroblastic Tumor with ALK mutation. Intern Med 2019;58:1029-32.

7. Theilen TM, Soerensen J, Bochennek K, et al. Crizotinib in $\mathrm{ALK}(+)$ inflammatory myofibroblastic tumors-Current experience and future perspectives. Pediatr Blood Cancer 2018. doi: 10.1002/pbc.26920.

8. Telugu RB, Prabhu AJ, Kalappurayil NB, et al. Clinicopathological Study of 18 Cases of Inflammatory Myofibroblastic Tumors with Reference to ALK-1 Expression: 5-Year Experience in a Tertiary Care Center. J Pathol Transl Med 2017;51:255-63.

9. Yu L, Liu J, Lao IW, et al. Epithelioid inflammatory myofibroblastic sarcoma: a clinicopathological, immunohistochemical and molecular cytogenetic analysis of five additional cases and review of the literature. Diagn Pathol 2016;11:67.
10. Berghmans T, Dusart M, Paesmans M, et al. Primary tumor standardized uptake value (SUVmax) measured on fluorodeoxyglucose positron emission tomography (FDGPET) is of prognostic value for survival in non-small cell lung cancer (NSCLC): a systematic review and metaanalysis (MA) by the European Lung Cancer Working Party for the IASLC Lung Cancer Staging Project. J Thorac Oncol 2008;3:6-12.

11. Ito K, Shimoji K, Miyata Y, et al. Prognostic value of posttreatment (18)F-FDG PET/CT for advanced head and neck cancer after combined intra-arterial chemotherapy and radiotherapy. Chin J Cancer Res 2014;26:30-7.

12. Kunkel M, Moergel M, Stockinger M, et al. Overexpression of GLUT-1 is associated with resistance to radiotherapy and adverse prognosis in squamous cell carcinoma of the oral cavity. Oral Oncol 2007;43:796-803.

13. Srinivas KS, Arunan M, Venkatachalapathy E, et al. The Prognostic Role of Maximum Standardized Uptake Value of 18 F-FlouroDeoxy Glucose Positron Emission Tomography-Computed Tomography in Head and Neck Cancer Patients Undergoing Chemoradiotherapy. J Int Soc Prev Community Dent 2019;9:159-65.

14. Sarmiento DE, Clevenger JA, Masters GA, et al. Epithelioid inflammatory myofibroblastic sarcoma: a case report. J Thorac Dis 2015;7:E513-6.

15. Dai Y, Wei Q, Schwager C, et al. Synergistic effects of crizotinib and radiotherapy in experimental EML4ALK fusion positive lung cancer. Radiother Oncol 2015;114:173-81.

16. Sun Y, Nowak KA, Zaorsky NG, et al. ALK inhibitor PF02341066 (crizotinib) increases sensitivity to radiation in non-small cell lung cancer expressing EML4-ALK. Mol Cancer Ther 2013;12:696-704.

17. FDA. FDA Approves Crizotinib Capsules.

doi: $10.21037 /$ tro.2020.04.01

Cite this article as: Lee CY, Hsu YL, Lee MY, Fang CY, Chen CC, Huang HY, Liu YR. Clinical significance of recombinant RANBP2-ALK oncogene to radiotherapy in a case of locally advanced rectal epithelioid inflammatory myofibroblastic sarcoma: case report and review of literature. Ther Radiol Oncol 2020;4:9. 